\title{
Ten-year experiences with Tetanus at a Tertiary hospital in Northwestern Tanzania: A retrospective review of 102 cases
}

Phillipo L Chalya ${ }^{1 *}$, Joseph B Mabula ${ }^{1 \dagger}$, Ramesh M Dass ${ }^{2 \dagger}$, Nkinda Mbelenge ${ }^{2 \dagger}$, Stephen E Mshana ${ }^{3+}$ and Japhet M Gilyoma'

\begin{abstract}
Background: Tetanus is still a major health problem in developing countries and it is associated with a high morbidity and mortality rate. There is paucity of published data regarding the management of tetanus in Tanzania, especially the study area. This study was conducted to describe our own experiences with tetanus outlining the clinical characteristics and treatment outcome of tetanus patients in our environment and to identify predictors of outcome of these patients.

Methods: This was a ten-year period retrospective study of patients who presented with a clinical diagnosis of tetanus at Bugando Medical Centre between January 2001 and December 2010. Data was analyzed using SPSS computer software system.

Results: A total of 102 patients were studied. The male to female ratio was 11.8: 1. The majority of patients (74.5\%) were aged $<40$ years and $51.0 \%$ of them were farmers. Only $23.5 \%$ of patients had prior tetanus immunization. $53.5 \%$ of patients had a reasonably identifiable acute injury prior to the onset of tetanus and commonly involved the lower limbs (53.8\%). The majority of patients (97.1\%) had generalized tetanus. The mean incubation period and period of onset were $8.62 \pm 4.34$ and $3.8 \pm 2.2$ days respectively. Complication rate was $54.9 \%$. The average overall duration of hospitalization was $34.12 \pm 38.44$ days (1-120 days). Mortality rate was $43.1 \%$. According to multivariate logistic regression analysis, the age $\geq 40$ years $(P=0.002)$, incubation period $<7$ days $(P=0.014)$, tracheostomy $(P=0.004)$, severity of tetanus $(P=$ $0.001)$ and need for ventilatory support $(P=0.013)$ were found to be significantly associated with higher mortality.

Conclusion: Tetanus remains a major public health problem in our centre and still carries unacceptably high morbidity and mortality despite the available advanced management facilities including ICU care. Young adult males are commonly affected. The incidence of tetanus can be reduced significantly by an effective immunization program and proper wound management of the patients. Early recognition, intense support and prompt treatment improves morbidity and mortality of patients diagnosed with tetanus.
\end{abstract}

Keywords: Tetanus, clinical characteristics, treatment outcome, predictors of outcome, Tanzania

\section{Background}

Tetanus, though a vaccine preventable disease, is still a significant public health problem throughout the world and it is associated with a high morbidity and mortality rate, particularly in the developing world [1-3]. The global incidence of tetanus is still estimated at one million

\footnotetext{
* Correspondence: drphillipoleo@yahoo.com

+ Contributed equally

'Department of Surgery, Weill-Bugando University College of Health

Sciences, Mwanza, Tanzania

Full list of author information is available at the end of the article
}

cases annually, with a case fatality ratio ranging from $6 \%$ to $72 \%$ depending on the availability of well equipped intensive care unit [3]. The incidence of tetanus in the developed world is markedly low and is no longer responsible for significant mortality, this has been attributed to high level of health awareness in terms of vaccination and availability of human and material resources to manage the disease [4]. In developed countries tetanus occurs mainly in elderly due to decline in protective antibodies [5,6] and in developing countries tetanus is common in the young due to lack of effective
C Biomed Central 
immunization program and appropriate treatment of injuries $[4,7]$.

Tetanus is caused by Clostridium Tetani, a gram positive, anaerobic and spore forming bacterium which is found in soil and in animal and human faeces and the usual mode of entry is through a punctured wounds or lacerations, although tetanus may follow surgery, burns, gangrene, chronic ulcers, dog bites, injections such as with drug users, dental infection, abortion and childbirth $[3,8]$. In some patients no portal of entry for the organism can be identified $[5,8]$.

Tetanus occurs sporadically and almost always affects non-immunized, partially immunized, or fully immunized persons who fail to maintain adequate immunity with booster doses of vaccine $[9,10]$. It is therefore very important, in order to have protection against tetanus, that all age groups have the universal primary immunization with subsequent maintenance of adequate antitoxin levels by means of appropriately timed boosters $[4,10]$.

The clinical manifestations of tetanus which include trismus (lockjaw), dysphagia, neck stiffness and generalized muscular rigidity is due to a powerful neurotoxin (Tetanospasmin) elaborated by the causative bacterium [11]. Four clinical forms of tetanus are recognized and they include generalized, localized, cephalic and neonatal tetanus [9-11]. Spasm related respiratory compromise, hospital acquired pneumonia and autonomic instability are usually the main causes of morbidity and mortality of this disease $[11,12]$. The diagnosis of tetanus is most frequently made on clinical manifestations, rather than on bacteriologic findings [8-13].

Management of tetanus patients is too demanding, prolonged, and expensive both in terms of materials and manpower $[4,14]$. A way to alleviate these problems is by adopting a rigorous tetanus immunization discipline in our community.

In Tanzania, like in most developing countries in the world, tetanus is endemic and remains an important health problem especially among the rural farming folks [4]. Tetanus is one of the most common causes of intensive care unit (ICU) admissions at Bugando Medical Centre (a tertiary hospital in Northwestern Tanzania\} and is associated with high morbidity and mortality. This study was undertaken to describe the clinical characteristics and treatment outcome of tetanus patients in our environment and to identify predictors of outcome among these patients.

\section{Methods}

\section{Study setting and design}

This was a ten-year period retrospective study of patients who presented with tetanus at Bugando Medical Centre between January 2001 and December 2010.
Bugando Medical Centre (BMC) is tertiary and teaching hospital for the Weill-Bugando University College of Health Sciences (WBUCHS) and is found in Mwanza city along the shore of Lake Victoria in northwestern Tanzania. It is a 1000-bedded hospital and serves approximately 13 million people from its neighboring regions namely Mwanza, Mara, Kagera, Shinyanga, Kigoma and Tabora. The hospital has a 12-bed adult and 10-bed paediatric multi-disciplinary Intensive Care Unit (ICU) which is headed by a consultant anesthesiologist and run by trained ICU nurses. Facilities in the unit include multi-parameter patient monitors, 1 defibrillator, syringe pumps, 2 mechanical ventilators and a standby anesthetic machine for emergency resuscitation when required. Oxygen supply is from oxygen concentrators available in the unit and cylinders provided on request from an oxygen bank in the hospital. The unit has one computer for accurate record keeping and documentation. Tetanus patients are usually admitted in the ICU and treated as per standard protocol for the management of tetanus.

\section{Study subjects}

The study included all patients of all age groups and gender who presented with a clinical diagnosis of tetanus. Patients who had incomplete or missed basic information were excluded from the study. The diagnosis of tetanus was wholly clinical and based on the presence of one or more of the following:-

1. Trismus

2. Rigidity of the neck and or abdomen

3. Reflex spasms

Tetanus was classified into generalized, cephalic, localized and neonatal types. Patients with rigidity and/or spasm limited to the wound bearing area of the body were classified as having localized tetanus, whereas those with trismus and generalized rigidity with or without generalized spasms were classified as having generalized tetanus. Tetanus occurring during neonatal period was classified as neonatal tetanus. A form of localized tetanus restricted to head and neck was classified as cephalic tetanus. The severity of tetanus was classified into mild, moderate severe and very severe according to the system reported by Ablett [15]. The treatment was started immediately once the diagnosis was made. The three objectives of therapy i.e. supportive care; neutralization of circulating toxin and removal (eradication) of the source of tetanospasmin (infected sites) was applied to all cases depending on the severity of spasms and availability of all essential facilities. The patients were treated as per standard protocol for the management of tetanus which included antibiotics (i.e. Penicillin, metranidazole or combination of both), wound care, passive immunization with human tetanus immune globulins 
(500 Units I/M stat) and active immunization with injection Tetanus Toxiod at the time of admission which was repeated when patient were discharged from the ward. The patients also received Diazepam for the control of spasm and mechanical ventilation when and where it was required. Details of demographic data (i.e. age, sex, occupation), tetanus immunization history, suspected portal of entry of infection, incubation time, clinical presentations, management, related complications, duration of intensive care unit admission, length of hospitalization, outcome and factors predicting the outcome were obtained from medical records and entered in a questionnaire before analysis. Incubation period was defined as the time from injury to the appearance of symptoms and the period of onset was defined as the interval between the first symptoms and the first spasm.

\section{Statistical analysis}

The statistical analysis was performed using statistical package for social sciences (SPSS) version 15.0 for Windows (SPSS, Chicago IL, U.S.A). The mean \pm standard deviation (SD), median and ranges were calculated for continuous variables whereas proportions and frequency tables were used to summarize categorical variables. Continuous variables were categorized. Chi-square $(\chi 2)$ test were used to test for the significance of association between the independent (predictor) and dependent (outcome) variables in the categorical variables. The level of significance was considered as $\mathrm{P}<0.05$. Multivariate logistic regression analysis was used to determine predictor variables that predict the outcome.

\section{Ethical consideration}

Ethical approval to conduct the study was sought from the WBUCHS/BMC joint institutional ethic review committee before the commencement of the study.

\section{Results}

One hundred-eighteen cases of tetanus were managed during the period under study. Of these, complete information was available on $102(86.4 \%)$ cases while there was some missing data on $16(13.6 \%)$ cases. Thus, a total of 102 patients were studied with an average of 10 cases per year (range of $8-14$ cases per year).

\section{Demographic data}

Males were 94 (92.2\%) and females were 8 (7.8\%) with a male to female ratio of 11.8: 1 . Their ages ranged from 8 to 72 years with a mean of $36.21 \pm 14.64$ years. The median was 34.00 years. The mean age of males and females was $35.14 \pm 14.82$ and $32.44 \pm 11.22$ years, respectively (P-value $>0.001)$. The modal age group was 31-40 years. Seventy-six $(74.5 \%)$ were below 40 years of age, while $26(25.5 \%)$ were aged 40 years and above. No cases of neonatal tetanus were reported. The majority of patients were farmers (51.0\%) (Table 1).

\section{Previous tetanus immunization history}

Previous tetanus immunization status was recorded in all patients. Of these, only twenty-four (23.5\%) patients had prior tetanus immunization, while the other seventy-eight $(76.5 \%)$ patients were not vaccinated or did not know their tetanus immunization status. However, in patients who had prior tetanus immunization there was no written proof of the immunization schedule in any cases. Serology test to detect anti-tetanus antibodies was not performed.

\section{Portals of entry and type of injury}

Acute injuries such us prick, puncture, laceration, burns were the most common portals of entry in $52.9 \%$ of cases and commonly occurred in the lower limbs $(53.8 \%)$. The portals of entry were not identified in $33.6 \%$ of cases (Table 1). Twenty-one (38.9\%) patients had medical wound care before hospital admission but none received tetanus immunoglobulin despite the absence of tetanus immunity.

\section{Incubation time and period of onset}

The incubation period, defined as the time between the inoculation of the wound and the onset of the symptoms, was known in eighty-eight (86.3\%) patients. The incubation period ranged from 3 to 26 days with a mean and median of $8.62 \pm 4.34$ and 7.8 days respectively. The majority of patients, $64(72.7 \%)$ had incubation period of less than 7 days and all of them had severe disease. The period of onset, defined as the interval between the first symptoms and the first spasm, was documented in 65 (63.7\%) and ranged from 2 to 9 days with the mean and median of $3.8 \pm 2.2$ days and 3.2 days respectively.

\section{Clinical presentation}

Ninety-nine (97.1\%) patients had generalized tetanus and the remaining two $(1.9 \%)$ and one $(0.9 \%)$ patients had cephalic and localized tetanus. respectively. No cases of neonatal tetanus were recorded. Assessment of severity according to Ablett classification system (Table 2 ) revealed that sixty-six $(64.8 \%)$ patients had severe disease. Eight (7.8\%) and four (3.9\%) patients had moderate and very severe disease. Assessment of severity was not recorded in twenty-four (23.5\%) patients.

Body stiffness/spasm (100\%), trismus (100\%) and dysphagia $(51.25 \%)$ were the three commonest presenting complaints (Table 3 ).

\section{The pattern of tetanus admission}

Eighty-four (82.4\%) patients were admitted in the ICU for isolation and ventilatory support and the remaining 
Table 1 Distribution of occupation and the portals of entry of tetanus

\begin{tabular}{|c|c|c|c|}
\hline Variable & Response & Number of patients & Percentage \\
\hline \multirow[t]{6}{*}{ Occupation } & Farmers & 52 & 51.0 \\
\hline & Labour/industrial workers & 22 & 21.6 \\
\hline & Civil servant/businessman & 6 & 5.8 \\
\hline & Housewives & 5 & 4.9 \\
\hline & Students & 5 & 4.9 \\
\hline & Unknown & 12 & 11.8 \\
\hline \multirow{10}{*}{ Portals of entry } & Acute injury (prick, puncture, laceration, burns) & 54 & 52.9 \\
\hline & Skin ulcers & 6 & 5.9 \\
\hline & Local surgical procedures & 3 & 2.9 \\
\hline & · Uvulectomy & 1 & \\
\hline & • Circumcision & 1 & \\
\hline & - Tooth extraction & 1 & \\
\hline & Chronic otitis media & 2 & 1.9 \\
\hline & Others (cellulitis/gangrene) & 2 & 1.9 \\
\hline & Abortion & 1 & 0.9 \\
\hline & No identified portal of entry & 34 & 33.6 \\
\hline \multirow[t]{6}{*}{ Anatomical site of the portal of entry } & Lower limbs & 55 & 53.8 \\
\hline & Upper limbs & 5 & 4.9 \\
\hline & Head/neck & 5 & 4.9 \\
\hline & Trunk & 2 & 1.9 \\
\hline & Genitalia & 1 & 0.9 \\
\hline & Unknown & 34 & 33.6 \\
\hline
\end{tabular}

eighteen $(17.6 \%)$ patients were admitted in isolation rooms in the general wards.

All the patients who were admitted in the ICU required ventilatory support. Mechanical ventilation was used in only $32(31.4 \%)$ cases. The average days on ventilatory support were 16.4 days (1-34 days). Of the patients who were admitted in the wards, $11(61.1 \%)$ patients were later transferred to ICU for ventilatory support and close monitoring.

\section{Treatment of patients}

Almost all patients were managed with tetanus toxoid, human tetanus immunoglobulin, antibiotic therapy (penicillin and metranidazole), wound care (wound toilet and debridement), muscle relaxants, sedatives, heparin prophylaxis and artificial ventilation. Tracheostomy was performed in 16 (15.7\%) patients and mechanical ventilation was used in $32(31.4 \%)$ cases. Supportive treatment such as balanced fluid and calorie intake, prevention of gastric stress ulcer, prevention of pressure sores were provided in all patients.

\section{Complications}

Complications of tetanus were documented in 56 (54.9\%) patients. These included respiratory complications (pneumonia, respiratory failure) in 18 (32.1\%) patients, cardiovascular (tachycardia, hypertension) in 11 (19.6\%), gastrointestinal complications (paralytic ileus) in $10(17.9 \%)$, renal complications (renal failure) in 4 (7.1\%), neurological complications (seizures) in 10 (17.9\%) and others in 3 (5.4\%).

\section{Outcome of tetanus patients}

Of the 102patients, 58 (56.9\%) patients were alive. of these, $53(91.4 \%)$ patients were discharged well and the remaining $5(8.6 \%)$ patients were discharged with

Table 2 Ablett Classification of the Severity of Tetanus [16]

\begin{tabular}{lll}
\hline Grade & Severity & Clinical features \\
\hline I & Mild & Mild to moderate trismus; general spasticity; no respiratory embarrassment; no spasms; little or no dysphagia \\
II & Moderate & Moderate trismus; well-marked rigidity; mild to moderate but short spasms; moderate respiratory embarrassment with an \\
& increased respiratory rate $>30$, mild dysphagia
\end{tabular}


Table 3 Clinical presentation of 102 tetanus patients

\begin{tabular}{lll}
\hline Clinical presentation & Number of patients & Percentages \\
\hline Body stiffness/spasm & 102 & 100 \\
Trismus & 102 & 100 \\
Dysphagia & 65 & 63.7 \\
Body aches & 24 & 23.5 \\
Backache & 12 & 11.8 \\
Fever & 11 & 10.8 \\
Headache & 9 & 8.8 \\
Abdominal pain & 8 & 7.8 \\
Jaw pain & 4 & 2.9 \\
Shortness of breath & 4 & 2.9 \\
Urinary retention & 2 & 1.9 \\
Chest pain & 2 & 1.9 \\
\hline
\end{tabular}

permanent disabilities such as persistent vegetative state due to hypoxic brain damage (2 patients), limb amputation ( 2 patients) and persistent abnormal gait in 1 patient. There were forty-four deaths, accounting for an overall mortality of $43.1 \%$ (Figure 1 ).

Majority of the deaths occurred in the first few days: 11 (25.0\%) died in the first 3days while 33 (75.0\%) \% died in the first 10 days. Of the patients who were discharged alive, only 17 (29.3\%) received tetanus toxiod before discharged. The predictors of mortality according to univariate and multivariate logistic regression analyses are shown in table 4.

Average ICU stay was 19.3 days (range 1-26 days) and the overall mean duration of hospital stay was $34.12 \pm$ 38.44 days (1-120 days). The median duration of hospitalization was 32.00 days. The mean and median duration of hospitalization for non-survivors were $6.2 \pm 4.8$ days (1-28 days) and 5.8 days respectively.

\section{Discussion}

Tetanus is still prevalent in developing countries and constitutes significantly to high morbidity and mortality despite the documented effectiveness of tetanus vaccines and its availability since 1923 [1-3]. High incidence of

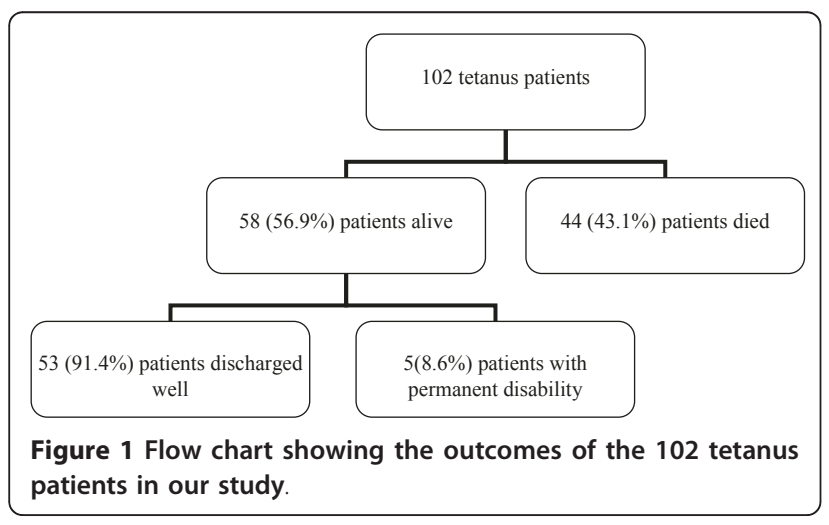

tetanus admissions in developing countries including Tanzania is attributed to low levels of health awareness in terms of vaccination and availability of human and material resources to manage the disease $[4,7]$. This observation is reflected in our study as more than three quarters of our patients were not vaccinated or did not know their tetanus immunization status. This finding calls for preventive measures to reduce the incidence of this disease, such as wide immunization coverage and health education.

In agreement with other studies in developing countries $[4,13,14,16]$, tetanus patients in the present study were quite young which is in contrast to other studies in developed countries $[8,9]$. This observation can be explained by the fact that in developing countries tetanus is common in the young due to lack of effective immunization program and inappropriate treatment of injuries [4,7] whereas in developed countries tetanus occurs mainly in elderly due to decline in protective antibodies [5,6].

In this study, male patients were more affected than females. The male preponderance in this study has been reported elsewhere $[4,6,8,9,11,12]$. This could be explained by the fact that men tend to spend more time outdoor, in farming activities and other types of fieldwork. Hence, they are more likely to be exposed to both the causal organism, C. tetani, which is ubiquitous in soil in a tropical country like Tanzania and the penetrating injury necessary for the organism to enter the body. The high proportion of admission among males in this study also reflects the low vaccination rates among males in the community as compared to females and children who gets their vaccination during pregnancy and childhood respectively. Health education on the importance of vaccination among males is highly needed to prevent from contracting this serious disease.

Majority $(51.0 \%)$ of the tetanus patients in this study were farmers which is in agreement with other studies $[6,8]$. This observation is in contrast to a Nigerian study which reported students and civil servants as the majority of cases [16]. This pattern of occupational risk group in our study can be explained by the fact that farmers or the peoples who live in the rural areas and engage themselves in the agricultural sector are more likely to be exposed to the causal organism as well as the injury necessary for the organism to enter the body.

In agreement with other studies $[8,9,16,17]$, the most common portal of entry in this study was injuries in the lower limbs. This is in contrast to Joshi et al [12] who reported upper limbs as the most common portal of entry. This lower limb preponderance in this study could be explained by the fact that C. tetani exists in soil; hence, any lower limb injury would be open to contamination and infection by this organism, bearing in 
Table 4 The predictors of mortality according to univariate and multivariate logistic regression analyses

\begin{tabular}{|c|c|c|c|c|c|c|}
\hline \multirow[t]{2}{*}{ Predictor variable } & \multirow{2}{*}{$\begin{array}{l}\text { Number of survivors (N/ } \\
\%)\end{array}$} & \multirow{2}{*}{$\begin{array}{l}\text { Number of non-survivors ( } \mathrm{n} / \\
\%)\end{array}$} & \multicolumn{2}{|c|}{ Univariate analysis } & \multicolumn{2}{|c|}{ Multivariate analysis } \\
\hline & & & $\begin{array}{l}\text { O.R. } \\
\text { (95\% C.I.) }\end{array}$ & $\begin{array}{l}\mathrm{P} \text { - } \\
\text { value }\end{array}$ & $\begin{array}{l}\text { O.R. } \\
\text { (95\% C.I.) }\end{array}$ & $\begin{array}{l}\mathrm{P} \text { - } \\
\text { value }\end{array}$ \\
\hline \multicolumn{7}{|l|}{ Age (years) } \\
\hline$<40$ & $56(73.7 \%)$ & $20(26.3 \%)$ & & & & \\
\hline$\geq 40$ & $2(7.7 \%)$ & $24(92.3 \%)$ & $3.4(2.8-5.2)$ & 0.012 & $5.8(3.7-17.3)$ & 0.002 \\
\hline \multicolumn{7}{|l|}{ Sex } \\
\hline Male & $54(47.4 \%)$ & $40(42.6 \%)$ & & & & \\
\hline Female & $4(50.0 \%)$ & $4(50.0 \%)$ & $0.2(0.1-5.4)$ & 0.675 & $0.4(0.3-2.1)$ & 0.986 \\
\hline \multicolumn{7}{|c|}{$\begin{array}{l}\text { Incubation period(days) }(\mathrm{N}= \\
\text { 88) }\end{array}$} \\
\hline$<7$ & $24(37.5 \%)$ & $40(62.5 \%)$ & & & & \\
\hline$\geq 7$ & 20(83.3\%) & $4(16.7 \%)$ & $6.3(3.6-9.7)$ & 0.002 & $0.5(0.3-0.9)$ & 0.014 \\
\hline \multicolumn{7}{|c|}{$\begin{array}{l}\text { Period of onset (hours) }(\mathrm{N}= \\
65 \text { ) }\end{array}$} \\
\hline$<48$ & $11(29.7 \%)$ & $26(70.3 \%)$ & & & & \\
\hline$\geq 48$ & $10(35.7 \%)$ & $18(64.3 \%)$ & $0.4(0.2-2.6)$ & 0.561 & $1.7(0.5-3.2)$ & 0.937 \\
\hline \multicolumn{7}{|l|}{ Prior immunization } \\
\hline Yes & $16(66.7 \%)$ & $8(33.3 \%)$ & & & & \\
\hline No & $42(53.8 \%)$ & $36(46.2 \%)$ & $3.9(0.4-6.2$ & 0.068 & $0.9(0.5-2.5)$ & 0.561 \\
\hline \multicolumn{7}{|l|}{ Admission pattern } \\
\hline ICU & $48(57.1 \%)$ & $36(42.9 \%)$ & & & & \\
\hline Wards & $10(55.6 \%)$ & $8(44.4 \%)$ & $4.4(0.6-6.7)$ & 0.491 & $3.8(0.7-4.9)$ & 0.849 \\
\hline \multicolumn{7}{|l|}{ Type of tetanus } \\
\hline Generalized & $56(55.6 \%)$ & $43(43.4 \%)$ & & & & \\
\hline Cephalic & $1(50.0 \%)$ & $1(50.0 \%)$ & $2.5(0.9-3.1)$ & 0.067 & $1.7(0.2-5.4)$ & 0.082 \\
\hline Localized & $1(100 \%)$ & - & & & & \\
\hline \multicolumn{7}{|c|}{ Severity of tetanus ( $N=78$ ) } \\
\hline Moderate & $7(87.5 \%)$ & $1(12.5 \%)$ & & & & \\
\hline Severe & $27(38.6 \%)$ & $43(61.4 \%)$ & $2.4(1.3-6.3)$ & 0.012 & $4.7(2.5-9.1)$ & 0.001 \\
\hline \multicolumn{7}{|l|}{ Debridement done } \\
\hline Yes & $34(63.0 \%)$ & $20(37.0 \%)$ & & & & \\
\hline No & $24(50.0 \%)$ & $24(50.0 \%)$ & $2.4(0.6-3.9)$ & 0.075 & $5.1(0.9-6.8)$ & 0.089 \\
\hline \multicolumn{7}{|l|}{ Tracheostomy done } \\
\hline Yes & $14(87.5 \%)$ & $2(12.5 \%)$ & & & & \\
\hline No & $44(51.2 \%)$ & $42(48.8 \%)$ & $3.1(1.4-7.3)$ & 0.011 & $4.9(2.3-8.1)$ & 0.004 \\
\hline \multicolumn{7}{|c|}{ Need for ventilatory support } \\
\hline Yes & $26(81.3 \%)$ & $6(18.7 \%)$ & & & & \\
\hline No & $32(45.7 \%)$ & $38(54.3 \%)$ & $1.7(1.1-4.5)$ & 0.032 & $0.2(0.1-0.8)$ & 0.013 \\
\hline \multicolumn{7}{|l|}{ Complications } \\
\hline Present & $35(62.5 \%)$ & $21(37.5 \%)$ & & & & \\
\hline Absent & $23(50.0 \%)$ & $23(50.0 \%$ & $3,9(0.5-4.3)$ & 0.063 & $1.6(0.4-6.2)$ & 0.911 \\
\hline
\end{tabular}

mind too that most tetanus patients were rural farming folks. Also, the preponderance of lower limbs in our study is thought to result from poor protective footwear. The portals of entry were not identified in $33.6 \%$ of cases reflecting that the injuries were likely to be trivial to be recalled. Trivial wounds on the lower limbs as possible portals of entry for tetanus infection are common because most people in the rural areas do not wear shoes.
Body stiffness/spasm, trismus and dysphagia, in that order, were the commonest complaints of the tetanus patients in our series which is in agreement with other studies $[8,9,11,14]$. Hence, a high index of suspicion for tetanus is of paramount whenever patients present with any of these symptoms as tetanus is essentially a clinical diagnosis and laboratory results as well as cultures are of little diagnostic value [5]. If a patient presents with all the 
three complaints, the probability of tetanus would be extremely high.

The treatment of tetanus patients requires a well established intensive care facility with a medical and nursing staff experienced in treating artificially ventilated and haemodynamically unstable patients. The majority (82.4\%) of study patients required ICU management an observation which is also reported in other studies $[9,11]$. However, ICU admission in this study did not significantly improve the prognosis of these patients in terms of mortality. This may be attributed to low levels of tracheostomy and mechanical ventilation which were performed in only $15.7 \%$ and $31.4 \%$ of cases respectively.

In this study, tracheostomy to circumvent the problem of laryngeal spasm (which could lead to asphyxiation and hypoxia) and to enable tracheal suction and toilet to be carried out efficiently (airway protection) was performed in only $15.7 \%$ of patients which is similar to what was reported by Feroz and Rahman in Bangladesh [8]. This is in contrast with other studies which reported that all tetanus patients underwent tracheostomy $[9,18]$. The use of tracheostomy in the management of patients with severe tetanus will undoubtedly prevent death due to asphyxia from laryngeal muscle spasm (and acute airway obstruction), respiratory muscle spasm and aspiration [18]. The low rate of tracheostomy in our study may be responsible for high mortality rate among tetanus patients. There was no obvious explanation for the low rate of tracheostomy in this study.

Complication rate in the present study is high compared to other studies $[6,11]$. However, the presence of complication did not significantly affect the outcome of tetanus patients. Our complication pattern was fairly similar to what was reported by Feroz and Rahman in Bangladesh [8]. We could not find any obvious reason in literature to explain this similarity. Much attention must therefore be paid to prevent these complications through early diagnosis and management.

The prognosis of patients with tetanus has been reported variably. Overall, mortality is approximately $10-50 \%$, however in certain age groups e.g. neonates it is as high as $90-95 \%$ [19]. In this study, mortality rate was 43.1\% which is comparable with the observation reported by Mohammed et al [20], whereas Mchembe \& Mwafongo [4] in Tanzania and Zziwa [21] in Uganda have reported higher mortality rate of $72.7 \%$ and $47 \%$ respectively. The high mortality rate could be due to the gross inadequacy of human and material resources to manage severe tetanus in the intensive care unit, typical of developing countries like Tanzania [4,22]. Various factors have been known to affect the prognosis [11]. The poor prognostic factors in this study included age $\geq$ 40 years, shorter incubation periods $(<7$ days), low rate of tracheostomy, and severity of tetanus. Most of the deaths in our series were attributed to sudden cardiac arrest, respiratory failure and infective pulmonary complications, an observation similar to other studies $[8,21]$. In this study, only $29.3 \%$ of the patients who were discharged cured received tetanus toxiod before discharged a figure fairly consistent with that of other studies $[21,22]$. This finding calls for a need to provide health education on primary immunization and scheduled booster immunization that have greatly found to reduce the incidence of tetanus.

The overall mean duration of hospital stay in this study was $34.12 \pm 38.44$ days (1-120 days) which is high compared to other studies $[4,9,12,16,17]$. In one study, the overall mean duration of hospital stay was 83.0 days [8]. Prolonged duration of hospital stay has an impact on hospital resources as well as on increased cost of heath care, loss of productivity and reduced quality of life.

The potential limitation of this study is the fact that information about some patients was incomplete in view of the retrospective nature of the study. This might have introduced some bias in our findings.

\section{Conclusion}

Although tetanus is a vaccine preventable infectious disease, its prevalence is still high in our environment and it remains a difficult to treat disease with an unacceptably high morbidity and mortality rate, even with available advanced facilities for its management. Young adult males are commonly affected. The incidence of tetanus can be reduced significantly by an effective immunization program and proper wound management of the patients. Early recognition, intense support and prompt treatment improves morbidity and mortality of patients diagnosed with tetanus. Our study show comparable clinical pattern and outcome with other studies in the developing countries reported in the literatures.

\section{Acknowledgements}

We are grateful to the senior house officers in the department of Surgery for their support in data collection. We also like to thank all members of staff in Medical Record department for their cordial help during this study.

\section{Author details}

${ }^{1}$ Department of Surgery, Weill-Bugando University College of Health Sciences, Mwanza, Tanzania. ${ }^{2}$ Department of Orthopedics, Weill-Bugando University College of Health Sciences, Mwanza, Tanzania. ${ }^{3}$ Department of Microbiology, Weill-Bugando University College of Health Sciences, Mwanza, Tanzania.

\section{Authors' contributions}

PLC designed the study, contributed in literature search, data analysis, manuscript writing \& editing and submission of the manuscript. JBM, RMD, NM and SM participated in study design, data analysis, manuscript writing and editing JMG participated in study design, supervised the write up of the manuscript and edited the manuscript before submission. All the authors read and approved the final manuscript. 


\section{Competing interests}

The authors declare that they have no competing interests.

Received: 3 May 2011 Accepted: 8 July 2011 Published: 8 July 2011

\section{References}

1. Galazka A, Gasse F: The present status of tetanus and tetanus vaccination. Curr Top Microbial Immunol 1995, 195:31-53.

2. Anuradha S: Tetanus in adults-A continuing problem: An analysis of 217 patients over 3 years from Delhi, India, with special emphasis on predictors of mortality. Med J Malaysia 2006, 61(1):7-14.

3. Oladiran I, Meier DE, Ojelade AA, Olaolorun DA, Adeniran A, Tarpley JL: Tetanus continuing problem in the developing world. World J Surg 2002, 26(10):1282-85.

4. Mchembe MD, Mwafongo V: Tetanus and its treatment outcome in Dar es Salaam: need for male vaccination. East African Journal of Public Health 2005, , 2: 22-23.

5. Sandford JP: Tetanus-Forgotten but not gone. N Engl J Med 1995, 332:812-3.

6. Amare A1, Yami A: Case-fatality of adult Tetanus at Jimma University Teaching Hospital, Southwest Ethiopia. African Health Sciences 2011, 11(1):36-40

7. Dietz V, Milstien JB, van Loon F, Cochi S, Bennett J: Performance and potency of tetanus toxoid: implications for eliminating neonatal tetanus. Bull WHO 1996, 74:619-28.

8. Feroz AHM, Rahman MH: A Ten-year Retrospective Study of Tetanus at a Teaching hospital in Bangladesh. J Bangladesh Coll Phys Surg 2007, 25:62-69.

9. Lau LG, Kong KO, Chew PH: A ten-year retrospective study of tetanus at a general hospital in Malaysia. Singapore Med J 2001, 42(8):346-50.

10. Edlich RF, Hill LG, Mahler CA, Cox MJ, Becker DG, Horowitz JH: Management and prevention of tetanus. J Long Term Eff Med Implants 2003, 13(3):139-54

11. Younas NJ, Abro AH, Das K, Abdou AMS, Ustadi AM, Afzal S: Tetanus: Presentation and outcome in adults. Pak J Med Sci 2009, 25(5):760-765.

12. Joshi S, Agarwal B, Malla G, Karmacharya B: Complete elimination of tetanus is still elusive in developing countries: a review of adult tetanus cases from referral hospital in Eastern Nepal. Kathmandu Univ Med J (KUMJ) 2007, 5(3):378-81.

13. Adekanle O, Ayodeji OO, Olatunde LO: Tetanus in a Rural Setting of South-Western Nigeria: a Ten-Year Retrospective Study. Libyan I Med 2009, 4:100-4

14. Komolafe MA, Komolafe EO, Ogundare AO: Pattern and outcome of adult tetanus in Ile-Ife, Nigeria. Niger I Clin Prac 2007, 10(4):300-303.

15. Ablett J JL: Analysis and main experience in 82 patients treated in Leeds tetanus unit.Edited by: Ellis M. Symposium on tetanus in Great Britain. Leeds; 1967:1.

16. Chukwubike OA, God'spower AE: A 10-year review of outcome of management of tetanus in adults at a Nigerian tertiary hospital. Ann Afr Med 2009, 8(3):168-172.

17. Fawibe AE: The Pattern and Outcome of Adult Tetanus at a Sub-urban Tertiary Hospital in Nigeria. Journal of the College of Physicians and Surgeons Pakistan 2010, 20(1):68-70.

18. Fasunla AJ: Challenges of Tracheostomy in Patients Managed for Severe Tetanus in a Developing Country. Int J Prev Med 2010, 1(3):176-181.

19. Bhatia R, Parbharkar S, Grover VK: Tetanus. Neurol India 2002, 50:398-407.

20. Mohammed W, Bhojo AK, Nashaa T, Rohma S, Nadir AS, Aseem S: Autonomic nervous system dysfunction predicts poor prognosis in patients with mild to moderate tetanus. BMC Neurology 2005, 5:2.

21. Zziwa GB: Review of tetanus admissions to a rural Ugandan Hospital. UMU press; 2009:7(3):199-202.

22. Aboud S, Budha S, Othman MA: Tetanus at Mnazi Mmoja Hospital in Zanzibar, Tanzania. TMJ 2001, 16(3):5-7.

doi:10.1186/1749-7922-6-20

Cite this article as: Chalya et al:: Ten-year experiences with Tetanus at a Tertiary hospital in Northwestern Tanzania: A retrospective review of 102 cases. World Journal of Emergency Surgery 2011 6:20.

\section{Submit your next manuscript to BioMed Central and take full advantage of:}

- Convenient online submission

- Thorough peer review

- No space constraints or color figure charges

- Immediate publication on acceptance

- Inclusion in PubMed, CAS, Scopus and Google Scholar

- Research which is freely available for redistribution

Submit your manuscript at www.biomedcentral.com/submit 